\title{
Suppressive Influence of Time - Space White Noise on the Explosion of Solutions of Stochastic Fokker - Planck Delay Differential Equations
}

\author{
Augustine O. Atonuje and Jonathan Tsetimi \\ Department of Mathematics and Computer Science, Delta State University, Abraka, Nigeria.
}

Received: May 09, 2016 / Accepted: June 05, 2016 / Published: July 25, 2016.

\begin{abstract}
It is generally known that the solutions of deterministic and stochastic differential equations (SDEs) usually grow linearly at such a rate that they may become unbounded after a small lapse of time and may eventually blow up or explode in finite time. If the drift and diffusion functions are globally Lipschitz, linear growth may still be experienced, as well as a possible blow-up of solutions in finite time. In this paper, a nonlinear scalar delay differential equation with a constant time lag is perturbed by a multiplicative Ito-type time - space white noise to form a stochastic Fokker-Planck delay differential equation. It is established that no explosion is possible in the presence of any intrinsically slow time - space white noise of Ito - type as manifested in the resulting stochastic Fokker- Planck delay differential equation. Time - space white noise has a role to play since the solution of the classical nonlinear equation without it still exhibits explosion.
\end{abstract}

Keywords: Explosion, non-linear, stochastic Fokker Planck delay differential equation, time - space white noise, finite time.

\section{Introduction}

A search through the extensive literature on the theory of differential equations reveals that a sufficient condition for existence and uniqueness of a solution to an ordinary differential equation (ODE), delay differential equations (DDEs) and stochastic differential equations (SDEs) as well as their delay counterparts is that the drift and the diffusion coefficients must satisfy the Lipschitz condition and perhaps the linear growth condition. See for instance Arnold (1974), Oksendal (1992), Mao (1997), etc. More specifically, consider the stochastic delay differential equation

$$
\left.\begin{array}{rl}
d x(t) & =f(t, x(t), x(t-r))+g(t, x(t), x(t-r)) d B(t) \\
x(t) & =\Psi(t), t \in[-r, 0]
\end{array}\right\}
$$

where $r$ is a constant time lag, $f: \Re \times \Re \rightarrow \mathfrak{R}$,

Corresponding author: Augustine O. Atonuje, Department of Mathematics and Computer Science, Delta State University, Abraka, Nigeria. E-mail: austino412@yahoo.com. $g: \mathfrak{R} \times \mathfrak{R} \rightarrow \mathfrak{R} \quad, \quad B(t) \quad$ is a one-dimensional Brownian motion defined on a complete probability space $\left(\Omega, F,\left\{F_{t}\right\}_{t \geq 0}, \mathrm{P}\right)$. with a filtration $\left\{F_{t}\right\}_{t \geq 0}$, which is right continuous and contains all $\mathrm{P}$-null sets.

Theorem 1:

Assume that there exist constants $C, K>0$ such that

(i) $|f(x, t)-f(y, t)|^{2} \vee|g(x, t)-g(y, t)|^{2} \leq C|x-y|$, for all $x$ y $\in \mathfrak{R}, t \in\left[t_{0}, T\right]$

(Global Lipschitz condition)

$$
\begin{aligned}
& \text { (ii) }|f(x, t)|^{2} \vee|g(x, t)|^{2} \leq K\left(1+|x|^{2}\right), \\
& \text { for all }(x, t) \in \mathfrak{R}^{d} \times\left[t_{0}, T\right]
\end{aligned}
$$

(Linear growth condition)

Then there exists a unique solution $x(t)$ to Eq. (1).

Proof: [See Mao (1997), Arnold (1974)]

A serious deficiency is that this solution may grow at such a rate that it may become unbounded after a 
small lapse of time. Consequently, the solution may explode or blow up. One may observe that linear growth conditions such as (ii) in Theorem 1 above are not required for existence and uniqueness of solutions of differential equations. However, their absence may result in the sample paths of the solution blowing up or exploding in a finite time. Even at that, the global linear growth condition such as (ii) bounds $\mathrm{f}$ and $\mathrm{g}$ uniformly with respect to $t \in\left[t_{0}, T\right]$ and allows at most a linear increase of these functions with respect to $x$. It is usual to request that the coefficients of a stochastic differential equation should only be locally Lipschitz. More specifically, a function $\delta: \mathfrak{R}^{m} \rightarrow \mathfrak{R}^{n}$ is said to be locally Lipschitz if for any integer $\mathrm{N}>0$, there exists a constant $K_{N}$ such that

$$
\begin{aligned}
& |\delta(x)-\delta(y)| \leq K_{N}|x-y|, \\
& \forall|x| \leq N \text { and }|y| \leq N .
\end{aligned}
$$

This request becomes necessary because globally Lipschitz functions grow linearly whereas; locally Lipschitz functions do not behave like the global ones and as such reduce the possibility of explosion in solutions of SDEs.

A lot of research articles have been written on the explosion or blow-up time attempting to provide answers to certain questions such as: "When does a solution explode? What is the rate at which solutions of dynamical systems explode? What are the sufficient conditions for explosion to occur? What is the distribution of explosion times? What are the conditions for continuity of explosion times?' See for instance the interesting works of Leon et al (2013), Groisman and Rossi (2007), Mackane (1969), Jordan (2008) and some of the references therein.

This recent attraction towards research interest in blow-up time of solutions of both ordinary and partial differential equations is as a result of the fact that blow-up time is applicable in different areas of life. For example, blow-up time is applied in fatigue cracking to detect fatigue failure in solid materials such as aluminum and steel. The explosion time is generally a random time depending on the particular sample path and corresponds to the time of ultimate damage or fatigue failure in the materials. [See Kloeden and Platen (1992), Sobezyk and Spencer (1992). For results on the distribution of explosion times of solutions of stochastic differential equations and convergence of explosion times, the reader is referred to the article of Leon et al (2013) and Groisman and Rossi (2007) which are referenced in this paper. The foundation work on attempting to test for explosion times of SDEs was carried out by Feller (1952). Later, Hasminskii (1960) extended the results of Feller.

Bai and Li (2011), stochastically perturbed a non-linear deterministic differential equation with two Brownian noise feedbacks and studied how the noise can reduce explosion in solution and ensure the exponential stability of the system. Appleby and Kelly (2004) studied a class of functional differential equations and analyzed the effects of noise perturbation in the prevention of explosion in solutions of such equations. Other beautiful works on explosion of solutions of differential equations include those of McCarthy (2011), Malolepszy and Okrasinski (2008), Nie and Mai (2007). From all these research efforts, one sees that not much attention has been directed towards the study of the contribution of Ito type noise perturbation to the explosion of solutions of stochastic delay differential equations (SDDEs) to the best of the author's knowledge.

The aim of this article is to study the effect of an Ito - type time - space white noise on the explosive behavior of solutions of a non- linear delay differential equation. The resulting system after perturbation is a stochastic Fokker - Planck delay differential equation. It is established that even if the solution of classical non-linear delay differential equation blows up, this can never happen in the presence of the Brownian noise in the new stochastic Fokker - Planck delay 

Planck Delay Differential Equations

differential equation so formed. We are of the brilliant hope that this article will add to the few already existing research efforts on explosion of solutions of differential equations in the English Language literature.

\section{Preliminaries and Supporting Results:}

The classical non-linear delay differential equation studied is of the form

$$
\left.\begin{array}{l}
x(t)=p(t, x(t))+q(t, x(t), x(t-r)), t \in[0, T] \\
x(t)=\varphi(t), t \in[-r, 0]
\end{array}\right\}
$$

where $p, q \in C((0, \infty),(0, \infty))$, The function $p$ and $q$ in Eq.(2) are locally Lipschitz continuous, that is, for any positive integers $N$ and $M$ respectively, there are constants $K_{N}$ and $H_{M}$ such that

$$
\left.\begin{array}{c}
d X(t)=[p(t, X(t))+q(t, X(t), X(t-r))] d t+\sigma z(X(t)) d B(t), t \geq 0 \\
X(t)=\varphi(t), t \in[-r, 0]
\end{array}\right\}
$$

Where

$p: \mathfrak{R} \times \mathfrak{R} \rightarrow \mathfrak{R}, q: \mathfrak{R} \times \mathfrak{R} \rightarrow \mathfrak{R}, z: \mathfrak{R} \times \mathfrak{R} \rightarrow \mathfrak{R}$

are locally Lipschitz continuous. Eq. (2) and Eq. (4) have the same initial function $\phi \in C([-r, 0], \mathfrak{R})$ which is an $F(t)$ - measurable random3 variable such that such that $\mathrm{E}\|\phi\|^{2}<\infty$. By $C([-r, 0], \mathfrak{R})$, we refer to a family or Banach space of continuous functions from $[-r, 0]$ to $\mathfrak{R}$ armed with the supremum norm $\|\lambda\|=\operatorname{Sup}_{s \in[-r, 0]}|\lambda(s)|$ such that $\lambda \in C([-r, 0], \mathfrak{R}), \quad\{B(t)\}_{t \geq 0}$ is a one - dimensional Brownian motion, which represents the time-space white noise of Ito-type and whose initial condition is 0 at time $t=0$ and characterized by

(i) $\mathrm{E}[B(t)]=0$, a.s.

(ii) Independent increment;

for $\left(s_{1}, t_{1}\right) \cap\left(s_{2}, t_{2}\right)=\varphi$,

$B\left(t_{1}\right)-B\left(s_{1}\right), B\left(t_{2}\right)-B\left(s_{2}\right)$ are independent.

(iii) $B(t)-B(s)$ is $N(0, t-s)$, for $0 \leq s \leq t$

$$
\left.\begin{array}{l}
|p(x)-p(y)| \leq K_{N}|x-y| \\
|q(x)-q(y)| \leq H_{M}|x-y|
\end{array}\right\}
$$

for all $|x| \leq N,|y| \leq N$ and $|x| \leq H,|y| \leq H, x, y \in \mathfrak{R}$ $r \in(0, \infty)$ is a constant time lag. The local Lipschitz continuity satisfied by $p\left(x_{0}\right)$ and $q\left(x_{0}, x_{r}\right)$ guarantees that there is a unique solution of Eq. (2) up to the explosion time. A time -space white noise of Ito-type is applied to stochastically perturb the nonlinear scalar system in Eq.(2) into a stochastic Fokker - Planck delay differential equation (SFPDDE) described by a state variable $X$ confined to some closed interval. These types of equations evolve according to the stochastic delay differential equation of the form

(iv) $\mathrm{E}(B(t) B(s))=t \wedge s$, where $t \wedge s=\min (t, s)$

(v) Suppose that $0 \equiv t_{0}^{n}<t_{1}^{n}<t_{2}^{n}<\ldots .<t_{n}^{n}$

is a partition of or sub-division of the interval $[0, t]$ with $\max \left[t_{j+1}^{n}-t_{j}^{n}\right] \rightarrow 0$ as $n \rightarrow \infty$. Then L.i.m. $n \rightarrow \infty \sum_{j=0}^{n-1}\left[B\left(t_{j+1}^{n}\right)-B\left(t_{j}^{n}\right)\right]=t$ where L.i.m. means limit in $L^{2}$ sense.

Reason: By the fact that $B(t)-B(s)$ is normally distributed with mean zero and variance $t-s$, we have that

$$
\begin{aligned}
& \mathrm{E}\left[B\left(t_{j+1}^{n}\right)-B\left(t_{j}^{n}\right)\right]^{2}=\left[t_{j+1}^{n}-t_{j}^{n}\right] \\
& \mathrm{E} \sum_{j=0}^{n-1}\left[B\left(t_{j+1}^{n}\right)-B\left(t_{j}^{n}\right)\right]^{2}=t
\end{aligned}
$$

As such it becomes sufficient to establish that

$$
\operatorname{Lim}_{n} \mathrm{E}\left(\sum_{j=0}^{n-1}\left[B\left(t_{j+1}^{n}\right)-B\left(t_{j}^{n}\right)\right]^{2}-t\right)^{2}=0
$$

which is true since 


$$
\begin{aligned}
& \sum_{j=0}^{n-1}\left[B\left(t_{j+1}^{n}\right)-B\left(t_{j}^{n}\right)\right]^{2}-t= \\
& \sum_{j=0}^{n-1}\left[B\left(t_{j+1}^{n}\right)-B\left(t_{j}^{n}\right)\right]^{2}-\left[t_{j+1}-t_{j}\right]
\end{aligned}
$$

Consequently,

$$
\begin{aligned}
\operatorname{Lim}_{n} \mathrm{E}\left(\sum_{j=0}^{n-1}\left[B\left(t_{j+1}^{n}\right)-B\left(t_{j}^{n}\right)\right]^{2}-t\right)^{2} \\
=\sum_{j=0}^{n-1}\left(\left[B\left(t_{j+1}^{n}\right)-B\left(t_{j}^{n}\right)\right]^{2}-\left[t_{j+1}-t_{j}\right]\right)^{2} \\
=\sum_{j=0}^{n-1}\left(\mathrm{E}\left[B\left(t_{j+1}^{n}\right)-B\left(t_{j}^{n}\right)\right]^{4}+\left[t_{j+1}^{(n)}-t_{j}\right]\right)^{2} \\
-2\left[t_{j+1}^{(n)}-t_{j}\right] \mathrm{E}\left[B\left(t_{j+1}^{n}\right)-B\left(t_{j}^{n}\right)\right]^{2} \\
=\sum_{j=0}^{n-1}\left(\left\{3 \mathrm{E}\left[B\left(t_{j+1}^{n}-B\left(t_{j}^{n}\right)\right)\right]^{2}\right\}^{2}-\left[t_{j+1}^{(n)}-t_{j}^{(n)}\right]^{2}\right)^{2} \\
=\sum_{j=0}^{n-1} 2\left[t_{j+1}^{(n)}-t_{j}^{(n)}\right]^{2} \\
\leq 2 \max _{j}\left[t_{j+1}^{(n)}-t_{j}^{(n)}\right] \sum_{j=0}^{n-1} 2\left[t_{j+1}^{(n)}-t_{j}^{(n)}\right] \\
=2 t \max _{j}\left[t_{j+1}^{(n)}-t_{j}^{(n)}\right] \rightarrow 0 . \text { as } n \rightarrow \infty
\end{aligned}
$$

$\sigma$ is a parameter which scales the noise amplitude. We mention here without alteration in meaning, unless otherwise specified, the use of $\left(\Omega, F,\{F(t)\}_{t \geq 0}, \mathrm{P}\right)$, a filtered probability space with filtration $\{F(t)\}_{t \geq 0}$, which is complete in the sense that it satisfies the usual conditions, that is, $\{F(t)\}_{t \geq 0}$ is right continuous and each $\{F(t)\}_{t \geq 0}$ contains all p-null sets in $F$.

Definition 1:

A real - valued stochastic process $\{X(t)\}_{t \geq 0}$ is called a strong solution of Eq. (4) if it is a measurable, sample path continuous process such that it satisfies Eq. (4) and its initial condition. The solution is unique if there exists another indistinguishable solution $\{Y(t)\}_{t \geq 0}$ such that $\mathrm{P}[X(t)=Y(t), 0 \leq t \leq T]=1$.

Definition 2:

A solution $\{X(t)\}_{t \geq 0}$ of the Fokker Planck delay differential Eq. (4), which is defined on the interval $[0, T)$, grows at a specified rate and obeys $\operatorname{Lim}_{t \rightarrow \infty} X(t)=+\infty$ is said to explode or blow up in a finite time $\tau$, which depends on the initial datum, if its sample path $|X(t, w)| \rightarrow \infty$ as $t \rightarrow \tau\left(X_{0}, w\right)$ or equivalently, if $\operatorname{Lim}_{t \rightarrow \tau^{-}} X(t)=+\infty$. Hence for the solution $\{X(t)\}_{t \geq 0}, \tau$ is called an explosion or blow- up time for $X$ if either $T=\infty$ or $\operatorname{Lim}_{t \rightarrow T^{-}}|X(t)|=\infty$.

The Fokker - Planck delayed differential equation without the instantaneous functional in the drift term is more generally represented in the form:

$$
\left.\begin{array}{c}
d X(t)=q(t, X(t), X(t-r)) d t+\sigma z(X(t)) d B(t), t \geq 0 \\
X(t)=\phi(t), t \in[-r, 0]
\end{array}\right\}
$$

From Eq. (5), the probability transition function $\mathrm{P}$ is defined by two partial differential equations. Renowned mathematicians, Fokker, Planck and later Kolmogorov showed that for $x, y \in \mathfrak{R}^{n}, t>s$,

$$
\begin{aligned}
\frac{\delta \mathrm{P}(x, t / y, s)}{\delta t} & =\frac{\sigma^{2} \delta^{2} \mathrm{P}}{2 \delta x^{2}}-\frac{\delta}{\delta x}\left(b_{1}(x) \mathrm{P}\right) \\
& =\frac{\delta^{2}\left(b_{2}(x) \mathrm{P}\right)}{\delta x^{2}}=L_{x} \mathrm{P}(x, t / y, s)
\end{aligned}
$$

Eq. (4), Eq. (5) and Eq. (6) are generally called the Fokker - Planck equations. In application, in order to understand and predict events resulting from large fluctuations useful for describing changes of complicated disorder in biological and physical systems, the Fokker- Planck equation is one of the acceptable systems used in accounting for small fluctuations in stochastic dynamical systems.

Consider the nonlinear scalar differential equation of the form

$$
\left.\begin{array}{l}
x^{\prime}(t)=p(x(t)), t>0 \\
x(t)=\varphi>0
\end{array}\right\}
$$


It has been established (See Groisman and Rossi (2007) and Leon et al (2013)) that necessary and sufficient condition for the existence of a finite time explosion or blow up of solutions to Eq. (7) is

$$
\int_{1}^{\infty} \frac{1}{p(s)} d s<\infty
$$

Consider a more general nonlinear differential equation with a constant time lag

$$
\left.\begin{array}{l}
x(t)=p(x(t))+q(x(t-r)), t \geq 0 \\
x(t)=\phi(t), t \in[-r, 0]
\end{array}\right\}
$$

where $p, q \in C\left(\mathfrak{R}_{+}, \mathfrak{R}_{+}\right)$are locally Lipschitz continuous functions and the initial function $\phi \in C([-r, 0],(0, \infty))$. It can be shown that the condition in Eq. (8) is also necessary and sufficient for the blow up of the solution of Eq. (9) at a finite time. The following is a special case of the result found in McCarthy (2011) Theorem 2.2.1:

Theorem 2:

Assume that $p$ is locally Lipschitz continuous and satisfies $\int_{1}^{\infty} \frac{1}{p(s)} d s<\infty$. Let $q$ be locally Lipschitz continuous and $\phi \in C([-r, T],(0, \infty))$. Then there exists a finite time $T>0$ and some $x \in C([-r, T],(0, \infty))$ such that $x$ is monotone increasing on $[0, T] ; x$ is the unique solution of Eq. (9) and as such,

$$
\operatorname{Lim}_{t \rightarrow T^{-}} x(t)=\infty
$$

Proof:

By hypothesis, $x$ is continuous on $[-r, T]$ where $T \in(0, \infty)$ is such that $\operatorname{Lim}_{t \rightarrow T^{-}} x(t)=\infty$. Since, $p, q, \phi>0$ it follows that $x^{\mid}(t)>0$ for $t \in(0, T)$. We request that $\mathrm{T}$ which belongs to the time horizon $(0, \infty)=\mathfrak{R}_{+}$is such that $T \neq+\infty$.

Claim that the alternative $T=+\infty$ is true. Then one has that

$$
x^{\prime}(t)=>p(x(t)), t \geq 0
$$

Since $q>0$, and as such

$$
\operatorname{Lim}_{t \rightarrow T^{-}} x(t)=+\infty
$$

and

$$
T \leq \int_{\phi(0)}^{\infty} \frac{1}{p(s)} d s=\infty, t>0
$$

This contradicts the earlier assertion that $p(x(t))$ satisfies

$$
\int_{1}^{\infty} \frac{1}{p(s)} d s<\infty
$$

It must be that $\mathrm{T}$ is finite and since $p, q>0$, one gets that $\operatorname{Lim}_{t \rightarrow T^{-}} x(t)=+\infty$ where $\mathrm{T}$ is the blow - up time.

\section{Suppressive Influence of Time - Space White Noise:}

In this section, we study the contribution of multiplicative noise in suppressing the explosion of solution of the SFPDDE (4). In the main result, we establish that if there is at most a linear growth in the diffusion function under a certain condition on the noise scaling parameter and the instantaneous drift function, the presence of time -space multiplicative noise of Ito-type in the stochastic Fokker - Planck delay differential Eq. (4) suppresses the blow-up or explosion which might be present in the scalar non-stochastic delay differential Eq. (2).

Assumptions:

We consider system in Eq. (4) together with the following assumptions:

$$
\begin{aligned}
& H_{1}: r \in(0, \infty), \sigma=1 \\
& H_{2}: P: \Re \times \Re \rightarrow \Re, \\
& z: \Re \times \Re \rightarrow \Re \text { are locally Lipschitz continuous } \\
& H_{3}: \operatorname{Sup}_{X \in \Re} \frac{x p x-\frac{1}{2} z(x)^{2}}{1+|x|^{2}}<\infty \\
& H_{4} B(t) \text { is a semi-martingale, }
\end{aligned}
$$$$
\text { for all } t \geq 0 \text { up to time } \tau(x) \text {, the explosion time. }
$$ 


\section{Suppressive Influence of Time - Space White Noise on the Explosion of Solutions of Stochastic Fokker - 289 Planck Delay Differential Equations}

\section{Theorem 3:}

Assume that $\mathrm{H}_{3}$ holds and there $K>0$ such that $\mathrm{z}$ has at most a linear growth, that is,

$$
|z(x)| \leq k(1+|x|), x \in \mathfrak{R} .
$$

Furthermore, if $H_{1}, H_{2}$ and $H_{4}$ are satisfied, then the solution of the stochastic Fokker - Planck delay differential Eq. (4), can never explode or blow up at finite time.
Proof:

Claim that the general initial condition is uniformly bounded, that is, for $X(0)$, we let $\gamma_{M_{0}}=\left\{|X(0)| \leq M_{0}\right\}$. Introduce a sequence of stopping times by $\mu_{n-1}=\inf \left\{t>\eta_{n-1}:|X(t)|=R\right\}$ for some positive $R \geq M_{0} \quad$, where $\eta_{n}=\inf \left\{t>\mu_{n}:|X(t)|=R+1\right\} \quad$. Also let $\tau_{M_{0}}=\inf \left\{t>0: X(t)=M_{9}\right\}$. One gets

$$
X(t)=X(0)+\int_{0}^{t}[p(s, X(s))+q(s, X(s), X(s-r))] d s+\sigma \int_{0}^{t} z(s, X(s)) d B(s)
$$

and as such,

$$
\begin{aligned}
\mathrm{E}|X|_{\infty, \mu_{\tau} \wedge \tau_{M_{0}}}^{2} & \leq 2 \mathrm{E}|X(0)|^{2}+\mathrm{E} \int_{0}^{\mu_{\tau} \wedge \tau_{m_{0}}}[p(s, X(s))+q(s, X(s), X(s-r))] d s+K \mathrm{E} \int_{0}^{\mu_{\tau} \wedge \tau_{m_{0}}}\left\{1+|X(s)|^{2}\right\} d B(s) \\
& \leq 2 \mathrm{E}|X(0)|^{2}+\mathrm{E} \int_{0}^{\mu_{\tau} \wedge \tau_{m_{0}}}[p(s, X(s))+q(s, X(s), X(s-r))] d s+K T+\int_{0}^{T} \mathrm{E}|X|_{\infty, \mu_{\tau} \wedge \tau_{M_{0}}}^{2} d s
\end{aligned}
$$

Applying Gronwall's and noting that $H_{3}$ holds, we have

$$
\mathrm{E}|X|_{\infty, \mu_{\tau} \wedge \tau_{M_{0}}}^{2} \leq\left(2 \mathrm{E}|X((0))|^{2}+K T\right) e^{C T}+\int_{0}^{\mu_{\tau} \wedge \tau_{m_{0}}}[p(s, X(s))+q(s, X(s), X(s-r))] d s
$$

We now $M_{0} \rightarrow \infty$ which follows that $|X|_{\infty, \mu_{\tau} \wedge \tau(X)}<\infty$ and as such, $\mu_{\tau} \wedge \tau(X)<\tau(X)$. Again note that $\tau(X)=\infty$ results from $\mu_{\tau} \rightarrow \infty$ as $T \rightarrow \infty$. Therefore, solution of the stochastic Fokker - Planck delay differential equation (4) cannot explode.

\section{Final Remark:}

The crucial condition in theorem 3 which prevents explosion in Eq. (4) is $H_{3}$ in the set of assumptions. $\mathrm{H}_{3}$ ensures that the strength of the time-space white noise is large enough with respect to the non-delayed function $p$ in the drift term and at that instant, the explosion which is prevalent in the solution of the non-stochastic deterministic delay differential Eq. (2) can automatically be suppressed from occurring in Eq.

(4). This is as a result of the presence of noise.

\section{References}

[1] Arnold, L. (1974). Stochastic Differential Equations:
Theory and Applications. John Wiley \& Sons Inc. New York.

[2] Oksendal, B. (1992. Stochastic differential Equation: An introduction with Application. Springer- verlag, Berlin.

[3] Mao, X. (1997). Stochastic differential Equations and their Applications. Horwood Publishing Limited.

[4] Leon, J; Hernandez, L. P. and J. Villa-Morales (2013). On the distribution of Explosion time of stochastic differential Equations. Bol. Soc. Mat. Mexicana. 3, Vol. $19,125-138$.

[5] Griosman, P and Rossi, J. D. (2007). Explosion time in stochastic differential equations with small diffusion. Electronic Journal of Diffgerential Equations, Vol. 2007, No. 40, 1 - 9 .

[6] McKean, H. P. Jr. (1969). Stochastic Integrals. Academic Press New York.

[7] Jordan, P. M (2008). A note on Burgers Equation with time delay: Instability via finite time blow-up. Phys. Lett. A. 372, (42), $6363-6367$.

[8] Kloeden, P. E. and Platen, E. (1992). Numerial solution of stochastic differential Equations. Springer Verlag, Berlin.

[9] Sobezyk. K. and Spenser, B. F. (1992). Random fatigue. Academic Press Inc. Boston, MA.

[10] Feller, W. (1952). The parabolic Differential Equations 
290 Suppressive Influence of Time - Space White Noise on the Explosion of Solutions of Stochastic Fokker Planck Delay Differential Equations

and the Associated Semigroups of transformation, AM, 55, 468 - 519 .

[11] Hasminskii, R. Z. (1960). Ergodic Properties of Recurrent Diffusion Processes and stabilization of the solution of the Cauchy Problem for Parabolic Equations; TV, 5, 196 -214 .

[12] Bai, L. and Li, X. (2011). Analysis of Noise Under Regime Switching. Applied Mathematics, 2, 836 -842.

[13] Appleby, J. A. D. and Kelly, C. (2004). Prevention of Explosion in solutions of Functional Differential Equations by Noise Perturbation. Proceedings of Dynamic Systems and Applications, 4, 1 -12.
[14] McCarthy, M. J. (2011). Explosion and Unbounded Growth in nonlinear delay differential Equations: Numerical and Asymptotic Analysis, Ph. D. Thesis, School of Mathematical Sciences, Dublin City University.

[15] Malolepszy, T. and Okrasinski, W. (2008). Blow-up conditions for nonlinear Volterra Integral Equations with Power nonlinearity. Appl. Math. Lett. 21, (3), $307-$ 312.

[16] Nie, L. R. and Mei, D. C. (2007). Noise and time delay: Suppressed Population Explosion of the Mutualism System. Europhys. Lett. EPL, 79 (2); No. 2005, 6. 